\title{
Development of a model to estimate parameters in a snowpack based on capacitive measurements
}

\author{
Mercedes Noemi Murillo Abril $^{1} \quad$ Beathe Furenes $^{2} \quad$ Nils-Olav Skeie ${ }^{3}$ \\ ${ }^{1,3}$ Department of Electrical engineering, IT and Cybernetics, University of South-Eastern Norway, Norway, \\ mnmurillegmail.com; Nils-Olav@usn.no \\ ${ }^{2}$ Skagerak Energi, Norway, Beathe. Furenes@skagerakenergi.no
}

\begin{abstract}
In the optimization process of hydropower production, it is relevant to consider some information about the snowpack's parameters. Today, several techniques and devices to measure density, height, and snow water equivalent (SWE) in a snowpack. This paper discusses the development of linear regression models based on voltage measurements collected in a field test of a new measuring device that uses a vertical arrangement of capacitive sensors, to predict density, height, and SWE in a snowpack. The data collected grouped into six data sets and analyzed using the software for multivariable analysis Unscrambler X. From the results, three models were selected, one for each parameter. The models have a good prediction performance within the collection of samples. However, the model data sets used in the process do not have good representativity for other sampling conditions.
\end{abstract}

Keywords: $\quad$ snow density, capacitive sensor, model development, least square method

\section{Introduction}

\subsection{Background}

Hydropower is the main source of energy production in Norway (MPE 2019). Its production can be adjusted through the management of storage water in reservoirs (MPE, 2019). Therefore, having an accurate inflow forecast for the reservoirs optimize the energy's production. The inflow can vary drastically year to year, but it can be predicted through models that take in count the precipitations of the area. In Norway, approximately $30 \%$ of the annual precipitations falls as snow (Saloranta, 2014). The snow precipitations behave as unpredictable buffers of water. Depending on their melting process, they represent less or more water inflow to the reservoirs.

The inflow contribution of the snow buffers can be predicted through models. These models are in constant revision, and one of their sources of improvement is the measurement of snowpack's parameters (Saloranta, 2014). There are several measuring techniques and devices, some of them reviewed in (Kinar and Pomeroy, 2015). The master project reported in (Bjerke et al., 2019) proposes to design a new measuring device based on a vertical arrangement of fixed geometry capacitance plates. This concept, later implemented on a prototype described in a master thesis (Murillo Abril, 2020), required a model to traduce the sensors' measurements into the parameters of interest. These parameters are the snow density by layers, the total height of the snowpack, and its snow water equivalent (SWE).

This project aims to develop and evaluate three different linear regression models, analyze them, and choose the most suitable to calculate each parameter based on the measurements of the prototype.

\subsection{Scope and methods}

The focus of this paper is the models to calculate the snow density, the height, and the snow water equivalent (SWE) of a snowpack based on the measurements' values of the prototype, described by Murillo Abril (2020). Due to the warm weather on the zone $^{1}$ and mobility restrictions ${ }^{2}$, only one data set of samples was collected at the field test. From there, the samples were treated to derivate six data sets for the models' development.

The multivariate analysis software Unscrambler X 10.3 is used to analyze the data sets. The program has different linear regressions techniques and generates some plots which give a better understanding of the performance of the models. Therefore, the models' selection comes from the analysis of the program's calibration and validation results. The chosen models are simple mathematical relations that can be implemented in software, whether in the prototype's controller or an external system.

\footnotetext{
2 The Norwegian government imposed high fines to people staying outside their municipality (Forbes, 2020), due to the public health emergency of international concern of Covid-19, declared on the 30th of January, 2020 (WHO, 2020)

${ }^{1}$ There were low precipitations of snow in Porsgrunn area. Its lowest temperatures were around $-7^{\circ} \mathrm{C}(\mathrm{Yr}, 2020)$.
} 


\subsection{Outline of the paper}

Section 2 describes the relevant parts of the device used in the data collection process. Section 3 describes the data collection and their posterior treatment. Section 4 shows the data sets' analysis results, calibration, and validation of the models. The results are discussed in section 5, and some conclusions are drawn in Section 6.

\section{The device}

This section lists the device's features used for data collection during the development of the regression models.

\subsection{Concept of measurement}

The usage of a vertical arrangement of capacitors as a concept of measurement, discussed in (Bjerke et al., 2019), is based on the relation of the dielectric constant of the snow with its density and the fact that the snow does not accumulate with a uniform thickness (WSL, 2017). Figure 1 shows how the snow has different layers in a snowpack. It is possible to appreciate that the snow looks more compact in the lower layers than at the top. However, there are also intermediate layers more compacted than others on the bottom.

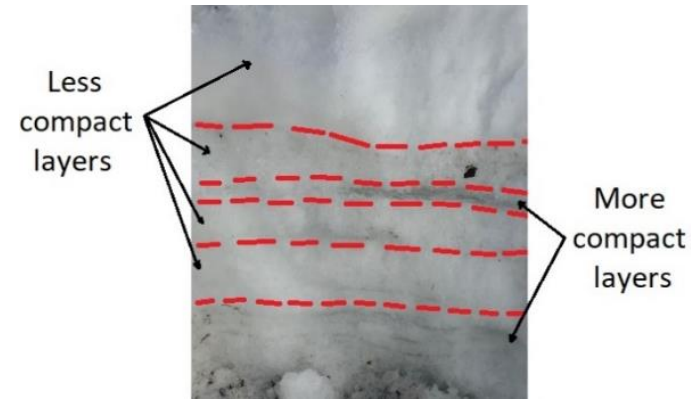

Figure 1: Example of different layers on a snowpack.

Figure 2 shows the sensing system implementing the concept of measuring mentioned earlier. The prototype uses a vertical arrangement of five capacitors installed in fixed distances. The sensor's positions, decided in cooperation with the customer, intent to cover a range of 1.20 meters of snow height with five sensors having a higher accuracy at the lowest layers. The prototype makes it possible to measure the density of up to five layers when the sensors are covered with snow. Because of the snow and air have different dielectric values (Evans, 1965), the prototype's sensors' values in contact with air are different from those covered in the snow. Consequently, the prototype is capable of sensing up to which height the sensors are covered. The SWE is a parameter related to the snow density and height (Brodzik, 2004). Consequently, it is assumed that as the system can recognize the density and height parameters, it can also collect different samples' values in association with the SWE of the snowpack.

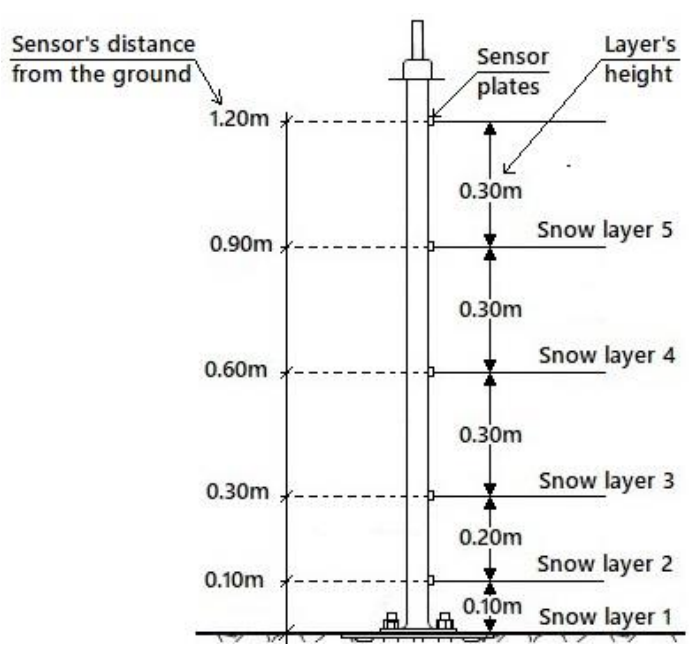

Figure 2: Sensing system of five capacitors.

\subsection{The capacitive sensor}

The sensor installed in the prototype is the Capacitive Soil Moisture Sensor v1.2. This sensor is typically used in garden applications to sense the humidity in the ground. The sensor has integrated a conducive and ground plates in a conditioning circuit. Figure 3 shows a picture of the installed sensor. The plates are covered by a corrosion-resistant material (How to Electronics, 2019), and act as capacitors of fixed geometry, varying their capacitance when surrounded by different mediums. The conditioning circuit uses a fixed frequency oscillator that feeds the plates with a square signal, and depending on its capacitance, the output signal's voltage varies (How to Electronics, 2019). For this prototype, the sensors are powered with 5 Volts DC, and their outputs are connected to the analog inputs of the Arduino Nano V3.0.

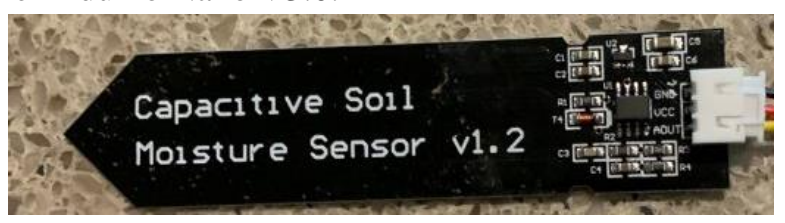

Figure 3: Capacitive sensor installed in the prototype (SwitchDoc, 2020).

The Arduino Nano is a small and complete board based on a microcontroller (Arduino, 2008). The microcontroller has eight analog inputs with an analogdigital converter (ADC) of 10 bits resolution (Circuits Today, 2020). The prototype uses the first five analog inputs to read the sensor's signals from 0 to 5 volts with a change of $4.88 \mathrm{mV}$.

\subsection{Construction and behavior}

The prototype, shown in Figure 4, uses drainpipes tubes and couplings to have a modular hollow structure. This structure allowed to place the sensors distanced, as shown in Figure 2 Inside the structure are the electronic circuits of the system, and the used material prevents the 
leaks of water when the device is covered by snow. On top of the sensors' column is the Arduino board placed.

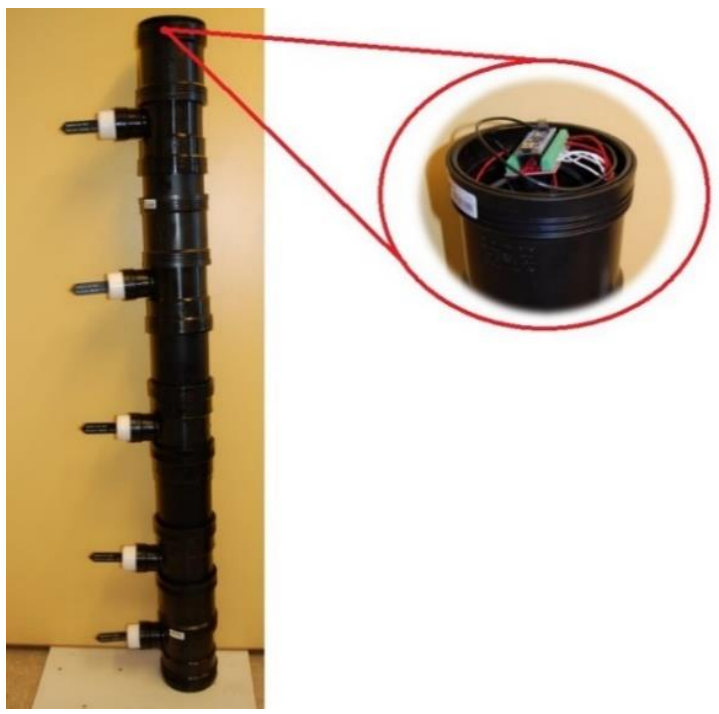

Figure 4: Prototype with five sensors at different heights and controller board on the top (Murillo Abril, 2020).

Figure 5 shows the flow of information on the system. The sensor senses the environment and conditions the output analog signals, as explained in the previous section. The microcontroller receives the signals through the ADC, processes and sends them through the serial port, incorporated on the board. To control the information flow in the prototype, the microcontroller's software has implemented an algorithm shown on the state diagram in Figure 6. The algorithm is designed to continuously read the system's sensors' values from the bottom to the top.

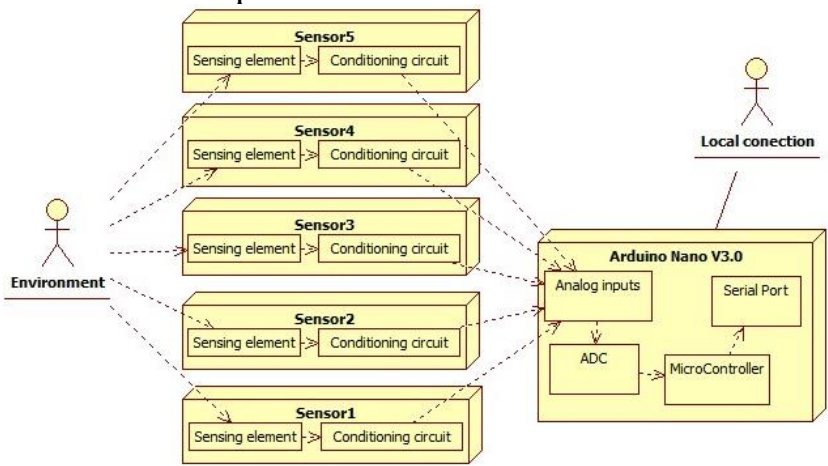

Figure 5: Diagram of the information flow through the prototype.

When the data collection process starts, the first state of the algorithm initializes a counter that identifies the sensor. It always begins from the lowest sensor first. In the next state, it reads the value of the sensor. Here a string variable is updated with the timestamp of the capture, the sensorID (sen+sensor number), and the captured value. The next state prints this variable in the serial port to be read in a serial monitor through a local connection.

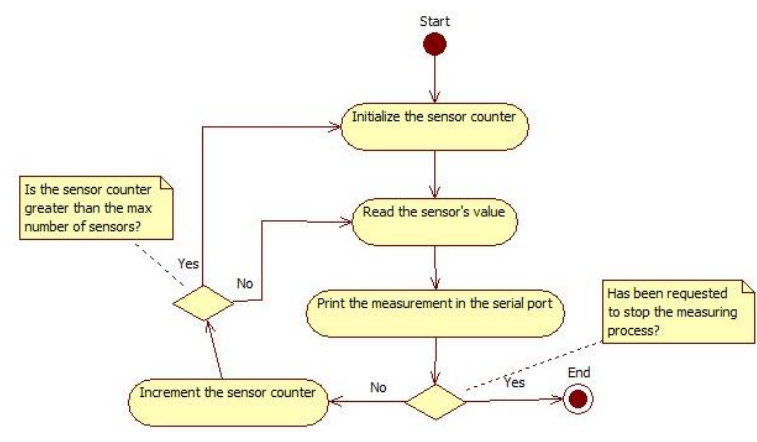

Figure 6: State diagram for the measuring process.

\section{Modelling data sets}

This section explains the collection and posterior treatment of the modeling data.

\subsection{Data collection}

The data collection took place in Viddaseter, one of the ski areas in Greenland (Visit Telemark, 2019). The location is about 500 hundred meters over the sea level. The field trip to collect the data was on the 26 of March 2020. The temperature of the day was $5.5^{\circ} \mathrm{C}$. The first step was to obtain a manual sample of snow density and height. The sample was taken using a cylindrical gauge and a marked metal stick, respectively. Around the manual sampling place, the terrain was prepared to dig a rectangle hole to have enough space for the prototype's installation, see Figure 7. The values of the density and height manually measured were 389.864 $\mathrm{Kg} / \mathrm{m}^{3}$ and 0.60 meters, respectively.

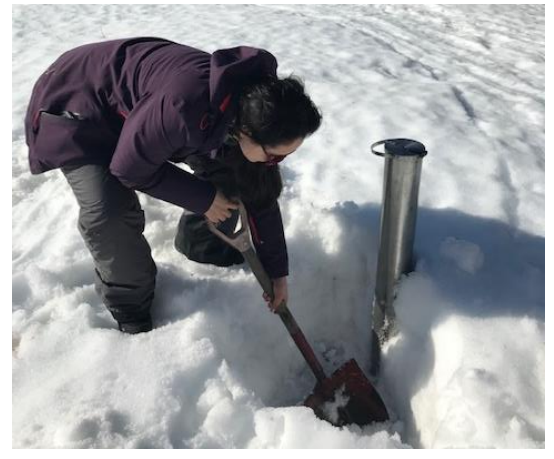

Figure 7: Preparation of the area to take the manual sample and later installation of the prototype.

In order to have more samples to train the model, the prototype was placed in two different places of the same pit. Figure 8 (left) shows the device placed on the first measuring place. Here the device was introduced on the snow. The first two sensors were fully covered on the snow, the third sensor was close to the top of the snow and the two remaining sensors were just surrounded by air. The second measuring place was in the opposite part of the pit. Figure 8(right) shows the device placed on the second point. Here the device was covered fully with snow up to the third sensor. The two remaining sensors on the top were surrounded by air. 


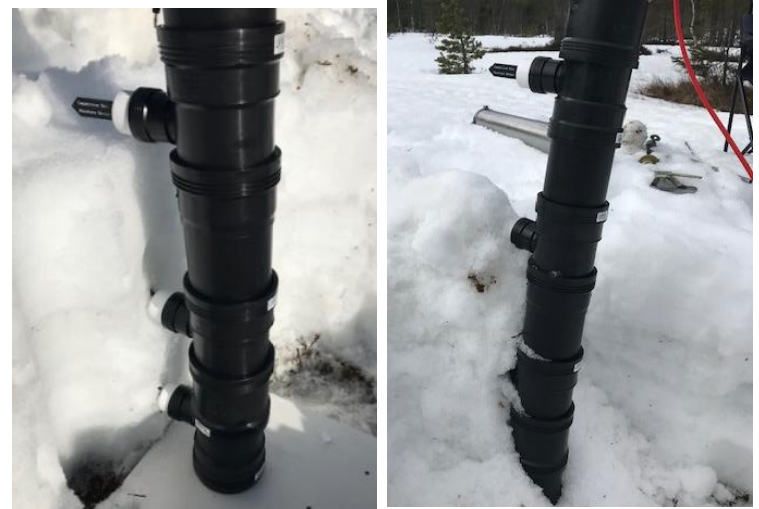

Figure 8: First measuring place (left), second measuring place (right).

To record the sensor's measurements' values, the system's controller had a local serial connection with an external computer, see Figure 9. The laptop had running the open software Tera Term, which is a serial terminal emulator. The emulator records the readings of the computer's serial port in a buffer. The measuring process started once the prototype was placed and stable in the measuring position. The process was run seven times. The first three tests were in the first measuring place. The tests 4, 5 and 7 in the second measuring place and test 6 all the sensors surrounded only by air. The mediums around the sensors during the tests are listed on Table 1. After each run the information on the buffer of Tera Term was saved into to a comma-separated values file.

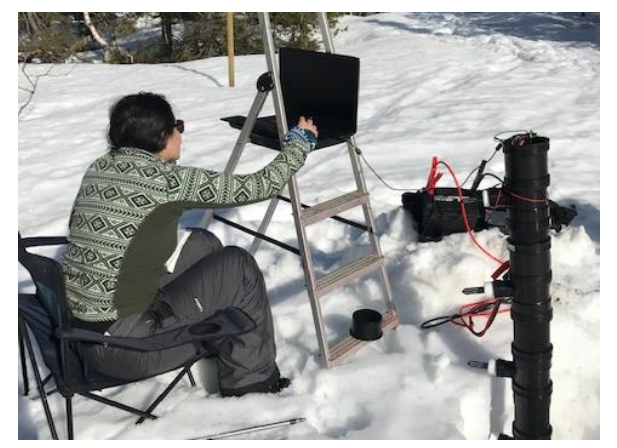

Figure 9: Data collection using an external computer in serial communication with the prototype.

Table 1: Conditions of the tests.

\begin{tabular}{|c|c|c|c|c|c|}
\hline Sensor & Sen1 & Sen2 & Sen3 & Sen4 & Sen5 \\
\hline 1 & snow & snow & air & air & air \\
\hline 2 & snow & snow & air & air & air \\
\hline 3 & snow & snow & air & air & air \\
\hline 4 & snow & snow & snow & air & air \\
\hline 5 & snow & snow & snow & air & air \\
\hline 6 & air & air & air & air & air \\
\hline 7 & snow & air & air & air & air \\
\hline
\end{tabular}

\subsection{Data treatment}

In a linear regression model, it is necessary to have a set of matched samples for the independent and dependent variables of the problem (Esbensen, 2010). The samples are used to calculate regression coefficients, which complete the general equation regression (Esbensen, 2010), presented in equation (1). In univariate regressions, the samples are divided into two matrices of one column, one containing all the values of the independent variable $(x)$ and the second one with all the dependent variable's values $(\mathrm{y})$.

$$
y=b_{0}+b_{1} x_{1}+b_{2} x_{2}+\cdots+b_{n} x_{n}
$$

In one case of the multivariate regressions, there is only one dependent variable, but there is more than one independent variable. In this case, the values of the independent variable are arranged on a matrix $(\mathrm{X})$ where each column corresponds to the values of each variable while the matrix of the dependent variable still is of one column. The values recorded during the field test of the prototype constitute raw measurements of the sensor's voltage. These are the independent variables of the regression, which has been arranged differently. The next three parts detail the arranging process.

\subsubsection{Pretreatment}

The samples from tests 1,2 , and 4 were not used for further analysis. They were measured using an old version of the controller's program, that did not work correctly. Before the regression matrices were constructed, the averages of the samples for each test were plotted, see Figure 10. The standard error (Francis, 2018) of the samples used to construct Figure 10 is 0.001 volts. It is possible to identify that the Test 3 values did not match the conditions of measurement stated in the third row of Table 1. In this test, the average for the sensors Sen 1 and Sen2 were expected to be close to the ones in Test 5, where these sensors are also covered with snow. However, their values are closer to the averages in Test 6 , where these sensors are exposed to air. Consequently, the only values used to develop the models were the ones from test 5 to 7 .

\section{Average of the values recorded the} 26-03-2020

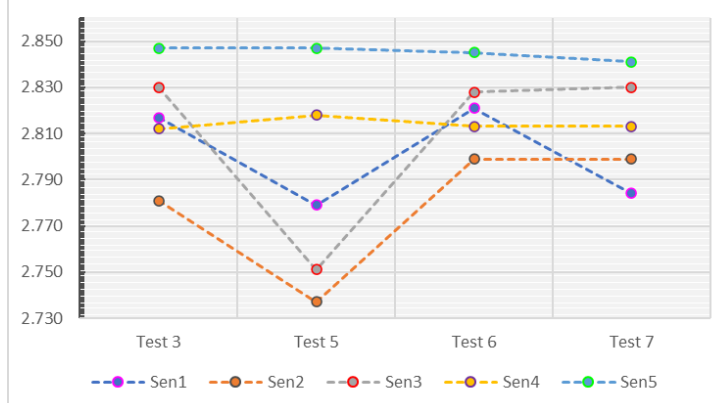

Figure 10: Average of the values by test run 3, 5, 6 and 7 . 


\subsubsection{Snow density}

According to the measuring concept, each sensor measures the density related to the material, which is in their surroundings. Therefore, the model to predict the snow density (dependent variable) requires the voltage value (independent variable) of the sensor in contact with the snow, as shown in equation (2). The $\mathrm{X}$ matrix consists of the record voltages of the sensors, from the tests mentioned before, stack on a column. There was only one manual sample for the density of the snow. Consequently, the Y matrix was completed with two o different values. When the sensor was covered by snow, the inserted value was the manual sample (389.864 $\mathrm{Kg} / \mathrm{m} 3$ ). When the sensor was only surrounded by air, the inserted value was $1.191 \mathrm{Kg} / \mathrm{m} 3$, which corresponds to the air's density at a pressure of $95276.7 \mathrm{~Pa}$ and a temperature of $5.5^{\circ} \mathrm{C}$ (Czenia and Haponiuk, 2020).

$$
\rho_{\text {snow }}=b_{0}-b_{1} V_{\text {sen }}
$$

These two columns of values form the Raw Data Set (SD). Applying a moving average filter to the measurements of the Raw Data Set (SD), two additional data set were created. The construction of the FilterX4 Data Set (SD) is shown in Figure 11. There the filter uses four values from the raw measurements. The same notion was used to create the FilterX8 Data Set (SD), but the filter used eight raw measurements. To the validation of the models, the technique used was the cross-validation. The constructed datasets are then divided into two parts. The first part used to calibrate the models and the second to validate them.

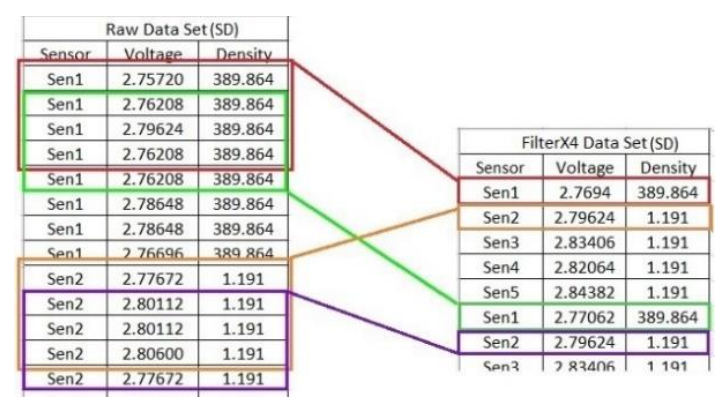

Figure 11: Construction of the FilterX4 Data Set (SD) from the Raw Data Set (SD) for calibration and validation of the Snow density model.

\subsubsection{Total height and SWE}

For the height and SWE parameters, the measurement concept is related to the number of sensors covered with snow during the sampling. Therefore, the construction of the matrix $\mathrm{X}$ for both cases was the same. This time, the sensors' values were independent variables, which their combination of values varies depending on the height and SWE of the surrounding. For the Y matrix, the inserted values vary depending on the parameter of interest. For the total height, the inserted values were corresponding to the measured height in the field. For the SWE, the inserted value depended on the number of layers' height cover by snow. The inserted value was calculated using the equation (3) (Murillo Abril, 2020). There, the terms of ' $z$ ' represent the height of the covered layers in meters. Consequently, the SWE values for tests 5, 6, and 7 were completed with 233.92; 0.00; and 38.99 meters.

$$
S W E=\left(z_{1}+z_{2}+\cdots+z_{n}\right) \frac{389.864}{100}
$$

Figure 12 partially shows the $\mathrm{X}$ and $\mathrm{Y}$ matrices used to form the height and SWE data sets. As in the previous part, the tests' measurements were stack to build a Raw Data Set $(\mathrm{H})$ and (SWE) for the height and SWE, respectively. Using the same filter technique, as for the density, over the values of each Raw Data Set created additional data set to analyze. For the height, the data sets used were Raw Data Set $(\mathrm{H})$, FilterX4 Data Set $(\mathrm{H})$, and FilterX8 Data Set $(\mathrm{H})$. While for the SWE, the data sets analyzed were Raw Data Set (SWE), FilterX4 Data Set (SWE) and FilterX8 Data Set (SWE). As in the previous section, the data sets are divided into two parts to the calibration and validation of the models.

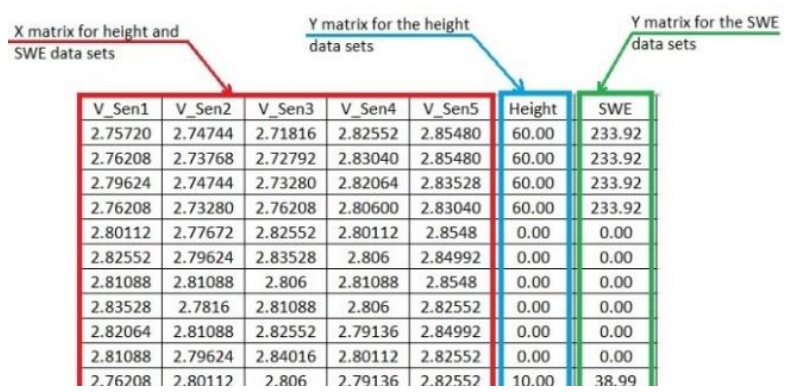

Figure 12: Partial $\mathrm{X}$ and $\mathrm{Y}$ matrices of raw data for height and SWE data sets.

\section{Results}

This section reports the results obtained from the calibration and validation processes of the models generated with the Unscrambler X 10.3.

\subsection{Snow density}

The snow density regression model corresponds to the analysis of univariate samples; therefore, it is only valid to calculate the snow's density by layers. In Unscrambler $\mathrm{X}$, the multivariate linear regression (MLR) was performed over the data sets but keeping the $\mathrm{X}$ matrix with only one column. This software then used the Least Square Regression (LS-R) method to minimize the total sum square of errors (SST) related to the data set. The SST value is calculated adding the square errors between the samples and a regression model as the average of the samples (Flotz, 2013). After finding the parameters of the calibrated models, the square sum of errors of the samples against the new regression models are calculated (SSE). The first two rows of Table 2 have the SST and SSE from the Unscrambler X analysis. Table 2 last row is the error reduction in percentage, and 
it was calculated from the ratio of the SSE and SST for each data set.

Table 2: SST, SSE and Error reduction (\%) for the Raw Data Set (SD), FilterX4 Data Set (SD) and FilterX8 Data Set (SD).

\begin{tabular}{|c|r|r|r|}
\hline $\begin{array}{c}\text { Data Set } \\
\text { Error }\end{array}$ & Raw $(S D)$ & \multicolumn{1}{|c|}{$\begin{array}{c}\text { FilterX4 } \\
(S D)\end{array}$} & $\begin{array}{c}\text { FilterX8 } \\
(S D)\end{array}$ \\
\hline SST & 16783250 & 14037500 & 10353740 \\
\hline SSE & 5083899 & 3462451 & 2489117 \\
\hline Error reduction & $30 \%$ & $25 \%$ & $24 \%$ \\
\hline
\end{tabular}

In all the cases, the SSE is reduced, so to the select one over the other two models, it was calculated the ration of reduction. In the same order as in Table 2, the errors were reduced to $30 \%, 25 \%$, and $24 \%$ of the original values. The model chosen was from the FilterX8 data set for reducing the most from the original error. The calibrated model is presented on the equation (4). There the $\rho_{\text {snow }}$ is the density in $\mathrm{Kg} / \mathrm{m} 3$ of the material in the surrounding of the sensor and $V_{\text {sen }}$ corresponds to the voltage measure by the sensor in Volts.

$$
\rho_{\text {snow }}=12565.58-4437.733 V_{\text {sen }}
$$

The model's performance to fairly predicts the density with new measurements was evaluated using the Predicted vs. Reference plot. The plot corresponding to the selected model is in Figure 13. The blue data corresponds to the evaluation using the calibration data set while the red corresponds to the evaluation using the reserved data set for the validation. The statistics, shown in the upper left corner of the plot, indicates that using the data that does not intervene in the model's calibration has the closest slope to the perfect prediction (1). The RMSE is high for both cases, but with the validation set is around 6 points lower.

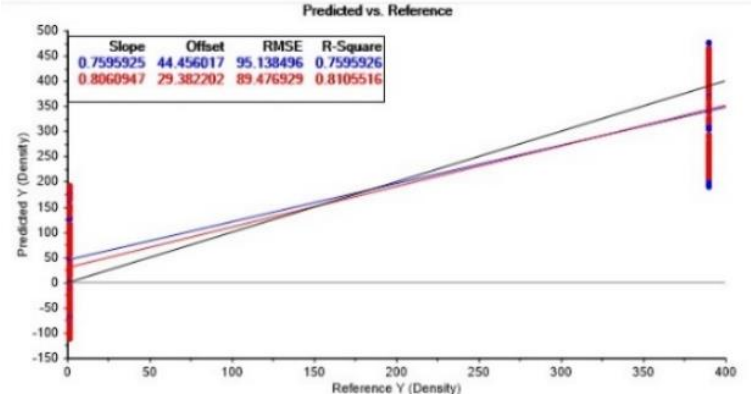

Figure 13: Predicted vs. Reference plot for the calibration and validation of the model from the FilterX8 Data Set (SD).

\subsection{Total height}

The samples for the height model are multivariate data sets. The Partial Least Square Regression (PLS-R) was run the Unscrambler $X 10.3$. The software decomposes the $X$ matrix generating several models using the decomposed factors for each data set and automatically choose the one that has explained the variance of the data set with the lowest numbers of factors. In this case, in the analysis of each data set, the software determined two-factor models. Manually the plots of Root Mean Square Error (RMSE) explained against the factors used in the results were examined to corroborate the software determination. To select one of the models, it was analyzed the Predicted vs. Reference plots for each data set. These plots are in Figure 14, Figure 15, and Figure 16, respectively. From those, the model from the FilterX8 data set has the lowest RMSE. Consequently, it is the chosen model, and its equation is (5). From this expression the $h$ represents the height of the snowpack in meters and the $V$ terms are the voltage values of the sensors in Volts.

$$
\begin{aligned}
h=2295.096- & 621.189 V_{\text {sen } 1}-326.097 V_{\text {sen } 2} \\
& -244.805 V_{\text {sen } 3}+311.173 V_{\text {sen } 4} \\
+ & 62.12442 V_{\text {sen } 5}
\end{aligned}
$$

As in the previous part, the Predicted vs. Reference plot also evaluates the models' performance to predict the height from new voltages' values. The chosen model has a slope of 0.97 , only three hundredths apart from the unit.

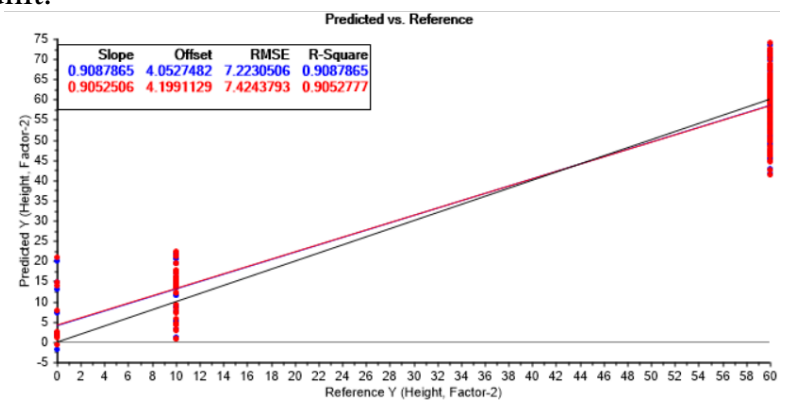

Figure 14: Predicted vs. Reference plot for the calibration and validation of the model from the Raw Data Set $(\mathrm{H})$.

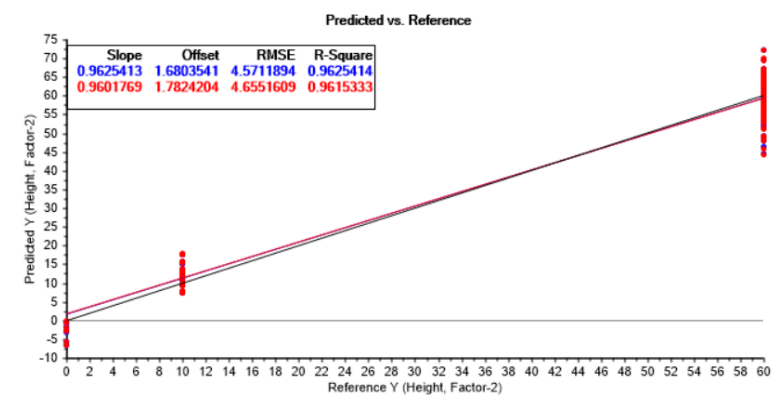

Figure 15:Predicted vs. Reference plot for the calibration and validation of the model from the FilterX4 Data Set $(\mathrm{H})$.

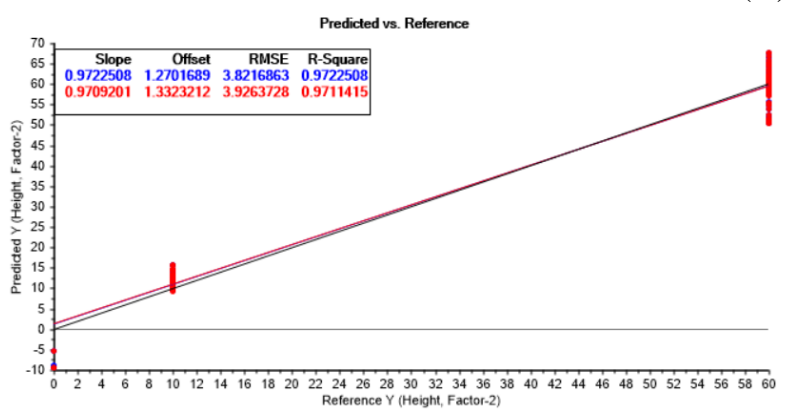

Figure 16: Predicted vs. Reference plot for the calibration and validation of the model from the FilterX8 Data Set $(\mathrm{H})$. 


\subsection{Snow water equivalent}

For the SWE model, the procedure used for the implementation in the height model was executed. The Predicted vs. Reference plots of the data sets are in Figure 17, Figure 18, and Figure 19, respectively. From the plots, the lowest RMSE corresponds to the FilterX8 data set. This is the model chosen and its equation is written in (6). The SWE is the parameter of interest given in millimeters and the $V$ terms are the voltage values of the sensors in Volts.

$$
\begin{aligned}
S W E=8947.771 & -2421.820 V_{\text {sen } 1} \\
& -1271.320 V_{\text {sen } 2}-954.394 V_{\text {sen } 3} \\
& +1213.319 V_{\text {sen } 4} \\
& +242.2006 V_{\text {sen } 5}
\end{aligned}
$$

The model's performance to predict the parameter from new measurement values, given by the slope in Figure 19 , is close to the unit.

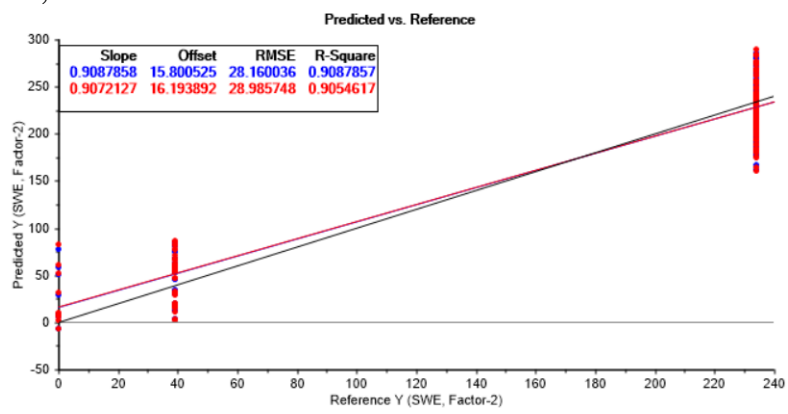

Figure 17: Predicted vs. Reference plot for the calibration and validation of the model from the Raw Data Set (SWE).

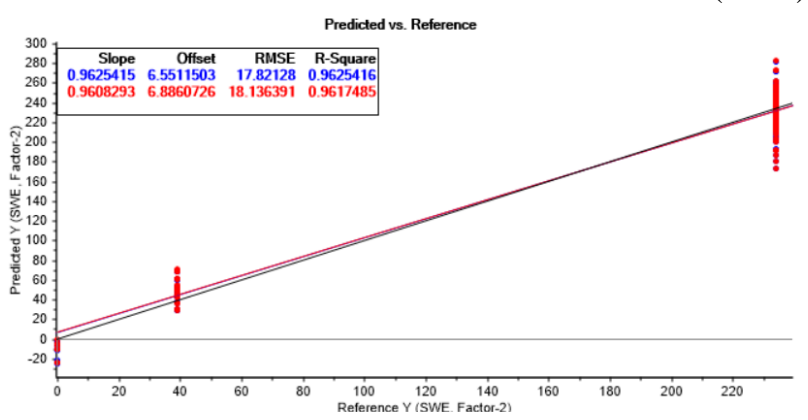

Figure 18: Predicted vs. Reference plot for the calibration and validation of the model from the FilterX4 Data Set (SWE).

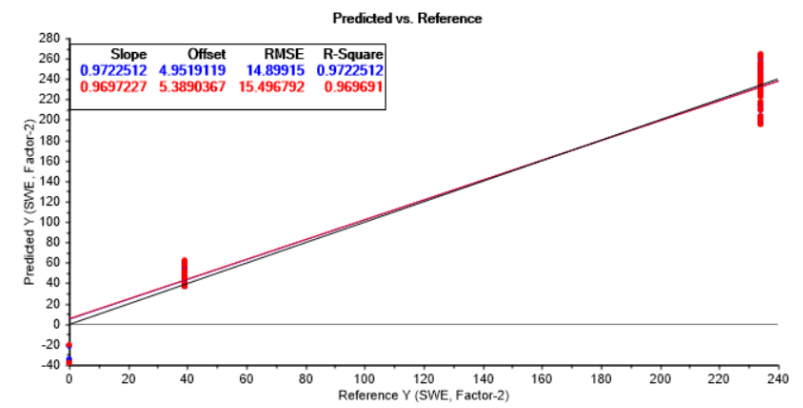

Figure 19: Predicted vs. Reference plot for the calibration and validation of the model from the FilterX8 Data Set (SWE).
A relevant result to include is the plot of the $\mathrm{X}$-Loading Weights, see Figure 20. In the analysis of the height and SWE models, the X-Loading Weights plot gave the same results. It shows a higher negative contribution from the values of the sensors 1 to 3 . The two remaining sensors have a lower positive contribution. This might be a consequence of the variation of snow coverage during the sampling process only on the three first sensors.

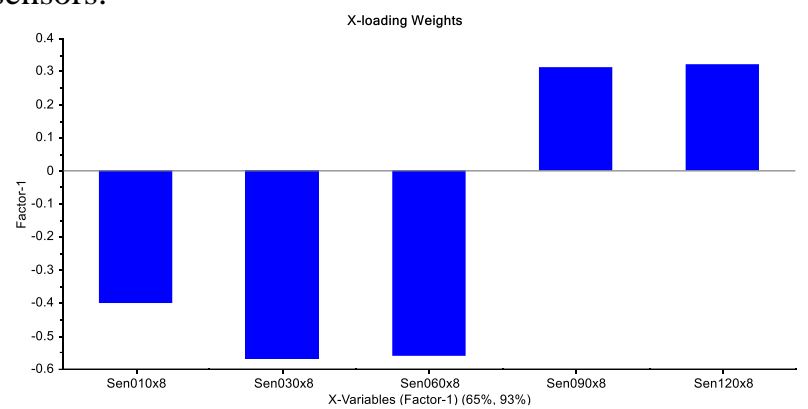

Figure 20: X-loading Weights plot for the analysis of the height and SWE models.

\section{Discussion}

In the development of regression models is important to have a rich data set of samples. Those must include as much information as possible in different measuring scenarios varying places, weather conditions, etc. However, due to the weather and mobility restrictions, the samples were collected in a single place without other variable conditions. Additionally, four of the seven test measurements were discarded due to errors during the measuring process. These two conditions make that the resulting models do not have a good prediction performance over other samplings scenarios.

In the models, the ones resulted from the FilterX8 Data Sets show better performance against the others. This can be the result that when the samples are filtered, noise errors are reduced in the data set. It is important to remark that the difference in performance between the models of the FilterX4 and FilterX8 data sets is not large. It is possible that increasing the values to use in the filter will increase the models' performance, but not a significant amount. Further works can test a model using 12 values in the filter to corroborate or discard the hypothesis. Also, it should analyze if the resources used to take more measurements and processes them contributes significantly to support a decision.

The slopes close to one in the Predicted vs. Reference plots indicate that the three chosen models' performances in the prediction of the parameters are close to the perfect performance. However, the confidence in these results cannot be high. The method used for the validation of the model was the crossvalidation. Even the validation of the models was done with a separate data set that did not intervene in the calibration process. It comes from a single sampling data set. It means that the models might be representing 
the errors associated with the sampling process more than the actual variance in the parameters of interest. Further work can include a new sampling data set to validate the models and revalidate its prediction performance.

The X-Loading Weights of the height and SWE models indicate that the principle of measurement can capture representative data to calculate the parameters of interest. A better structure of the sampling process can corroborate this theory and improve the models. A suggestion for the sampling process can include a step increase of the height of snow covering the sensors adding one at the time. For further works, the deployment of more than one device in different locations can increase the number of samples, and it can also improve the representativity of the data sets for calibrating and validating the models.

\section{Conclusion}

The Predicted vs. Reference plots in Figure 13, Figure 16, and Figure 19 show that the models generated have a good performance to predict the parameters of interest in the snowpack. However, this is only within the samples collected. The variance of the sensors' snow coverage during the sampling process, has a high impact in the loadings of the sensors' values for the height and SWE models. A manual measurement for the density that matches the positions of the sensors might introduce more important information to the sampling data sets.

\section{References}

Arduino. ARDUINO NANO: Overview. 2008. [Online]. Available: https://store.arduino.cc/arduino-nano. [Accessed 23 June 2020].

J. M. Bjerke, M. N. Murillo Abril, A. Jaganjac, and N. Pouladi. Measurement of snow density. Master's Project. University of South-Eastern Norway, Porsgrunn, Norway, 2019.

M. J. Brodzik. The relationship of snow water equivalent to snow depth and density. 2004. [Online]. Available: http://webarchiv.ethz.ch/arolla/Arolla_Data/SnowConditio ns/depth_to_swe.pdf. [Accessed 23 June 2020].

Circuits Today. Arduino Nano Tutorial - Pinout \& Schematics. 2020. [Online]. Available: https://www.circuitstoday.com/arduino-nano-tutorialpinout-schematics. [Accessed 23 June 2020].

D. Czernia and B. Haponiuk. Air Density Calculator. 2020. [Online]. Available: https://www.omnicalculator.com/physics/air-density. [Accessed 20 May 2020].

K. H. Esbensen. Multivariate Calibration (PCR/PLS). in Dominique Guyot, Frank Westad, and Lars P. Houmøller, editors, Multivariate data analysis in practice, pages 115154. CAMO Software AS, 2010. ISBN 82-993330-3-2.

S. Evans. Dielectric Properties of Ice and Snow-a Review. Journal of Glaciology, 5(42): 773-792, 1965. doi: 10.3189/S0022143000018840.

B. Flotz. Statistics 101: Linear Regression, the Very Basics. 2013. [Online]. Available:
https://www.youtube.com/watch?v=ZkjP5RJLQF4 [Accessed 21 May 2020].

Forbes. Norway Hands Out \$2,000 Fines Or Jail For Ignoring Coronavirus Quarantine. 2020. [Online]. Available: https://www.forbes.com/sites/davidnikel/2020/03/17/norw ay-hands-out-2000-fines-or-jail-for-ignoring-coronavirusquarantine/\#1bedeaf44f42. [Accessed 2 July 2020].

P. Francis. Standard Uncertainty, Standard error and Standard deviation. 2018. [Online]. Available: https://www.youtube.com/watch?v=NzrOfAsgxe8. [Accessed 04 September 2020].

How to Electronics. Interface Capacitive Soil Moisture Sensor v1.2 with Arduino. 2019. [Online]. Available: https://how2electronics.com/interface-capacitive-soilmoisture-sensorarduino/\#Capacitive_Soil_Moisture_Sensor_v12. [Accessed 19 May 2020].

N. J. Kinar and J. Pomeroy. Measurement of the physical properties of the snowpack. Reviews of Geophysics, 53(2): 481-544, 2015. doi: 10.1002/2015RG000481.

Ministry of Petroleum and Energy. Electricity Production. 2019. [Online]. Available: https://energifaktanorge.no/en/norskenergiforsyning/kraftproduksjon/. [Accessed 20 February 2020].

M. N. Murillo Abril. Development of a remote measurement. Master's thesis. University of South-Eastern Norway, Porsgrunn, Norway, 2020.

T. Saloranta. Report no. 6-2014, New version (v.1.1.1) of the seNorge snow model and snow maps for Norway. Norwegian Water Resources and Energy Directorate, Oslo, Norway 2014.

Swiss Federal Institute for Forest, Snow and Landscape Research WSL. Snowpack. 2017. [Online]. Available: https://www.slf.ch/en. [Accessed 21 February 2020].

SwitchDoc. Tutorial: Waterproofing your Capacitive Moisture Sensors. 2020. [Online]. Available: https://www.switchdoc.com/2020/07/tutorial-

waterproofing-capacitive-moisture-sensors/. [Accessed 4 September 2020].

Visit Telemark. Viddaseter. 2019. [Online]. Available: https://www.visittelemark.no/ting-a-gjore/viddaseterp502523. [Accessed 23 June 2020].

WHO. Rolling updates on coronavirus disease (COVID-19). $2020 . \quad$ [Online]. Available: https://www.who.int/emergencies/diseases/novelcoronavirus-2019/events-as-they-happen. [Accessed 10 May 2020].

Yr. Statistics - Porsgrunn. 2020. [Online]. Available: https://www.yr.no/en/statistics/graph/131680/Norway/Vestfold\%20og\%20Telemark/Porsgrunn/P orsgrunn?q=2020. [Accessed 2 July 2020]. 